\title{
Cortical Transplants Restore the Ability of Whisker Stimulation to Metabolically Activate Host Thalamus of Adult Rat with Parietal Cortical Lesions
}

\author{
Frank R. Sharp, Samuel Ciricillo and Manuel F. Gonzalez \\ Department of Neurology, University of California and VA Medical Center, \\ 4150 Clement St, San Francisco, CA 94121, USA
}

We have previously shown that fetal cortical transplants placed in lesion cavities in rat frontal cortex ameliorate the thalamic atrophy that occurs ipsilateral to such lesions. Moreover, the axotomized host brain thalamic neurons appear to sprout many fibers into the fetal cortical transplants. Lastly, cultured cortical and cerebellar neurons, but not glia, promote the survival of cultured thalamic neurons. In order to determine whether cortical transplants improve function of host thalamic neurons, the following studies were performed.

Whisker stimulation increased local cerebral glucose utilization (LCGU) in ipsilateral brainstem and in the contralateral ventroposteromedial (VPM) nucleus of the thalamus and whisker sensory cortex of normal adult rats. Though whisker stimulation increased LCGU in brainstem, it did not increase LCGU in the VPM of thalamus of rats that had their whisker sensory cortices removed 5 hours to 5 weeks previously. Since whisker stimulation did increase LCGU of the VPM following cortical tetrodotoxin (TTX) injections, the failure to activate the VPM following cortical ablations is probably not due to removal of cortical inputs to thalamus. In addition, thalamic neuronal death cannot explain the failure to activate VPM during whisker stimulation at 5 hours and one day following cortical lesions because the VPM thalamic neurons are known to be alive at these times. These results imply that activated brainstem neurons that project to the thalamus cannot metabolically activate axotomized VPM thalamic neurons. It is hypothesized that this occurs because of "stripping" of synaptic connections from brainstem neurons onto thalamic neurons by surrounding glia.

The thalamic neuronal death that occurs 7-14 days following cortical ablations was associated with thalamic hypometabolism. Since the excitotoxic thalamic cell death that occurs after cortical kainic acid injections is associated with thalamic hypermetabolism, it is proposed that the thalamic hypometabolism observed following cortical ablations is consistent with the concept that thalamic neurons die following cortical ablations because of the absence of a cortically-derived trophic factor. This is supported by further studies that show that transplantation of fetal cortex into cortical ablation sites increased LCGU in parts of the

TABLE

\begin{tabular}{lccc} 
& Lesion & Transplant \\
\hline VPM - L & $73 \pm 8^{*}$ & $123 \pm 25^{*} \#$ \\
& $\mathrm{R}$ & $101 \pm 10$ & $93 \pm 19$ \\
VPL - & $\mathrm{L}$ & $90 \pm 15$ & $93 \pm 25$ \\
& $\mathrm{R}$ & $104 \pm 11$ & $97 \pm 20$ \\
$\mathrm{LP}-$ & $\mathrm{L}$ & $76 \pm 11^{*}$ & $75 \pm 9 *$ \\
& $\mathrm{R}$ & $104 \pm 7$ & $107 \pm 23$ \\
\hline
\end{tabular}

LCGU ( $\mu$ moles glucose $/ 100 \mathrm{mg}$ brain/minute) in rats that had the left parietal cortex removed (Lesion) or had fetal cortex transplanted into left parietal cortex ablations (Transplants) 5 weeks previously. LCGU is listed for the left $(L)$ and right $(R)$ sides of the VPM, VPL and LP nuclei of the thalamus. *, L-R, \#, Lesion-Transplant, $\mathrm{p}<.05$ using Student's t-test. 
ipsilateral thalamus above the intact side. Moreover, double-label 2-deoxyglucose studies suggested that whisker stimulation could activate the host VPM of the thalamus in some transplanted subjects. It is suggested that: fetal cortical transplants provide a trophic factor to axotomized thalamic neurons; that the increased
LCGU of these neurons reflects increased protein synthetic activity; and that the cortical trophic factor facilitates continued contact of brainstem synapses on thalamic neurons so that whisker stimulation can activate the VPM of the thalamus in subjects with cortical transplants but not in subjects with cortical ablations. 

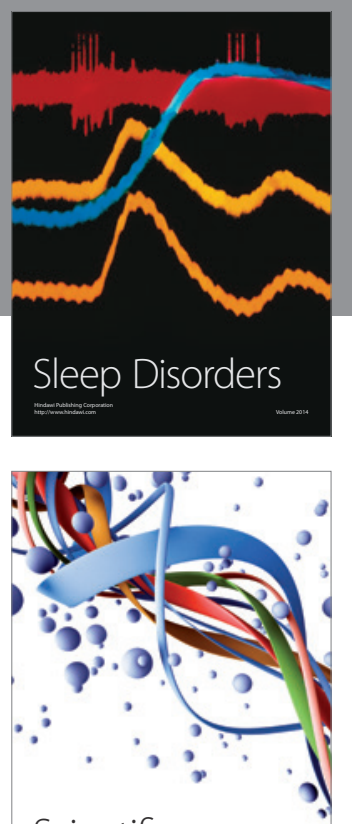

Scientifica
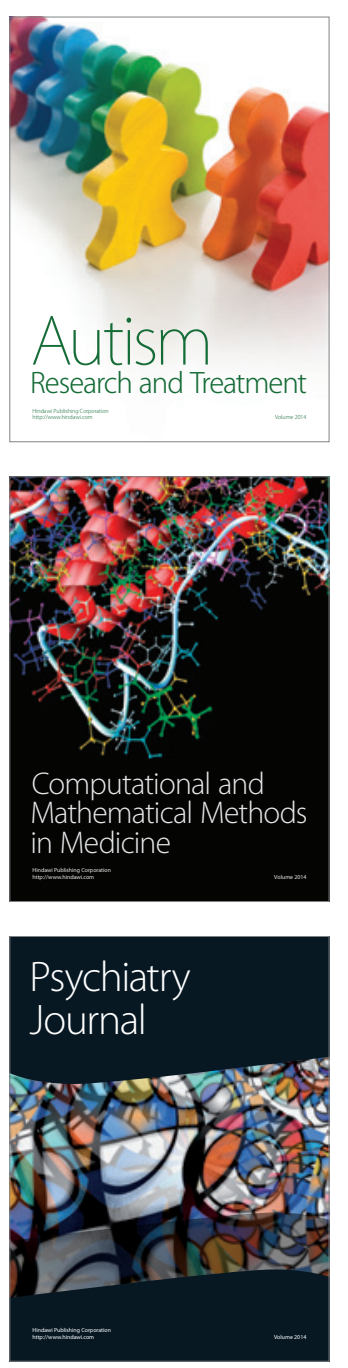
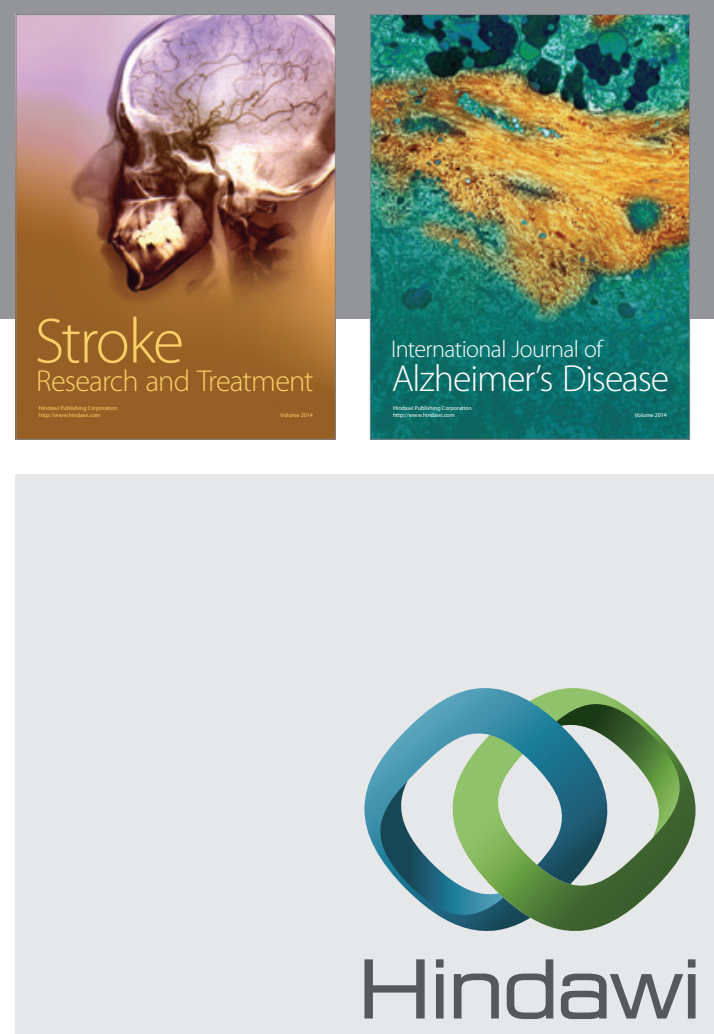

Submit your manuscripts at

http://www.hindawi.com
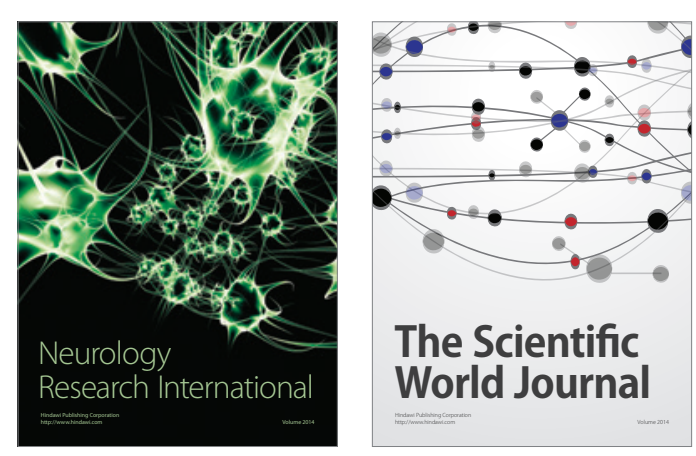

The Scientific World Journal

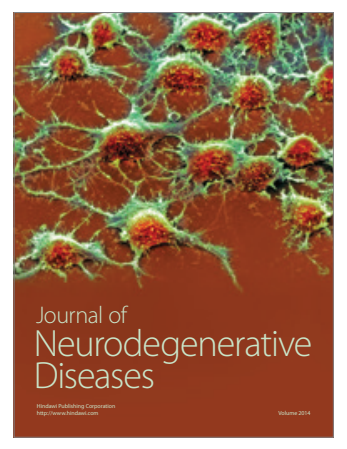

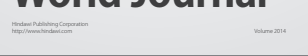

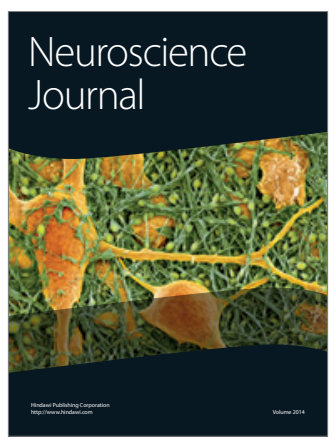

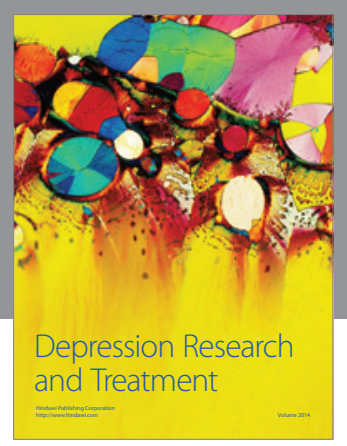
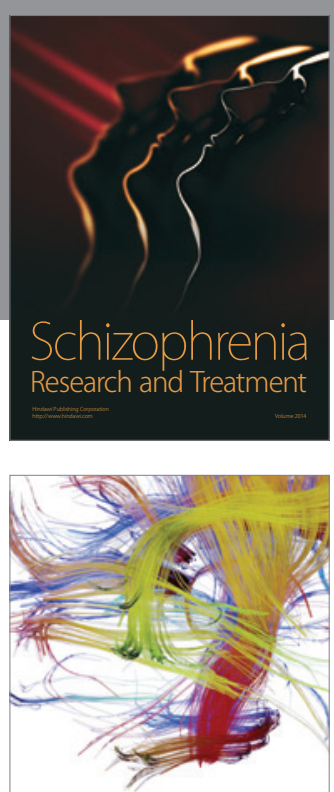

Brain Science

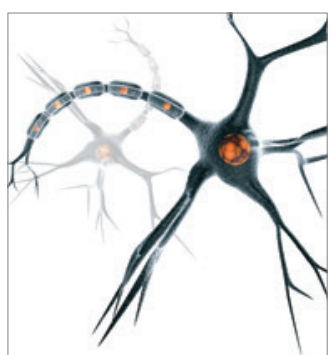

Neural Plasticity
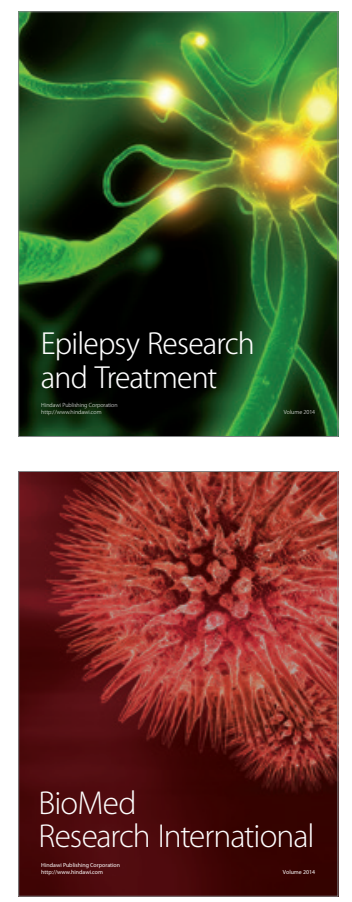

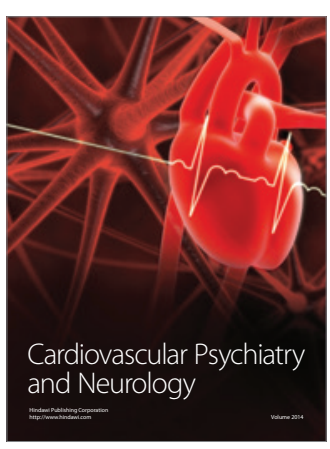

Parkinson's

Disease
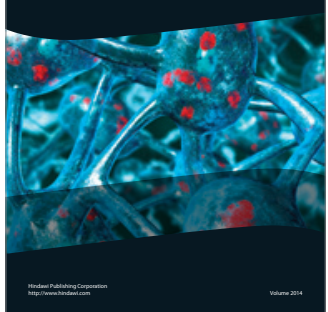